\title{
Agenesis of the Gallbladder Diagnosed by Magnetic Resonance Cholangiography: Report of a Case and Review of the Literature
}

\section{Oktay Yener, Mehmet Zeki Buldanlı, Hayati Eksioglu, Metin Leblebici, Orhan Alimoglu}

Faculty of Medicine, Istanbul Medeniyet University, Göztepe Training and Research Hospital, Istanbul, Turkey

Received October 31, 2014; Accepted March 12, 2015.

Key words: Agenesis - Gallbladder - Laparoscopy

Abstract: Gallbladder agenesis (GA) is a rare congenital anomaly of the biliary system often associated with other congenital abnormalities. Patients become symptomatic in $23 \%$ of cases (Richards et al., 1993). GA is often misinterpreted as other diseases, therefore, leading to unnecessary surgery. Many of these patients develop a typical symptomatology of cholelithiasis that leads them to operating theatre. If an operative procedure is done, it is better to remain at the level of laparoscopy because further surgical investigation may lead to detrimental biliary tract injuries (Waisberg et al., 2002). We present a case of GA, diagnosed by magnetic resonance with cholangiopancreatography.

Mailing Address: Dr. Oktay Yener, Faculty of Medicine, Istanbul Medeniyet University, Göztepe Training and Research Hospital, Plaj Yolu Kaya apt. 23/10, Caddebostan, Istanbul, Turkey; Mobile Phone: +905 337088 469; e-mail: oktayener@gmail.com 


\section{Introduction}

Gallbladder agenesis (GA) is a very rare anatomical abnormality first reported by Lemery in 1707 or by Bergman in 1702 (Wilson and Deitrick, 1986).

The incidence in the general population is reported as $13-65$ cases $/ 100000$ (Latimer et al., 1947). In clinical series, the incidence is $0.007-0.0027 \%$, while in autopsy series it is $0.04-0.13 \%$. GA may be associated with other system malformations as gastrointestinal, genitourinary, cardiovascular, musculoskeletal or some congenital syndromes. Once a surgeon meets such a situation, it is wiser to do nothing further in the operating theatre and to try to establish the accurate diagnosis postoperatively mainly by using MRCP (magnetic resonance cholangiopancreatography) (Adusumilli and Siegelman, 2002).

Patients become symptomatic in $23 \%$ of cases, and GA will almost always be misinterpreted as cholecystitis with cystic duct obstruction or as sclero-atrophic gallbladder, therefore, leading to unnecessary surgery (Malde, 2010).

It is difficult to establish a correct preoperative diagnosis of GA in symptomatic patients because of the nonspecific nature of the symptoms.

\section{Case report}

A 33-year-old female patient was admitted to clinic for upper abdominal pain, bloating, and dyspepsia for the last one month. Her blood pressure and pulse rate were regular and her body temperature was normal. Results of all hematological and biochemical investigations were within normal limits.

Ultrasonic investigation revealed a sclero-atrophic gallbladder with a normal biliary tree (Figure 1). According to these findings, it was decided to perform a laparoscopic cholecystectomy. On laparoscopy, the gallbladder could not be visualized from the junction of the left and right hepatic ducts until it disappeared behind the second part of the duodenum. We did not find any evidence of a

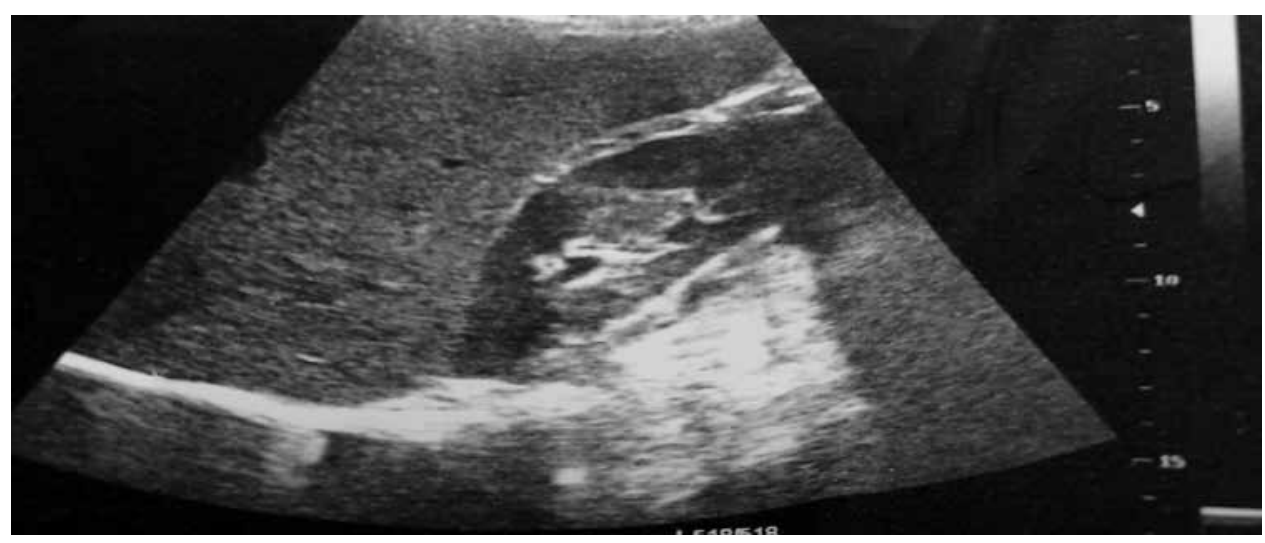

Figure 1 - Ultrasonic investigation revealed a sclero-atrophic gallbladder with a normal biliary tree. 


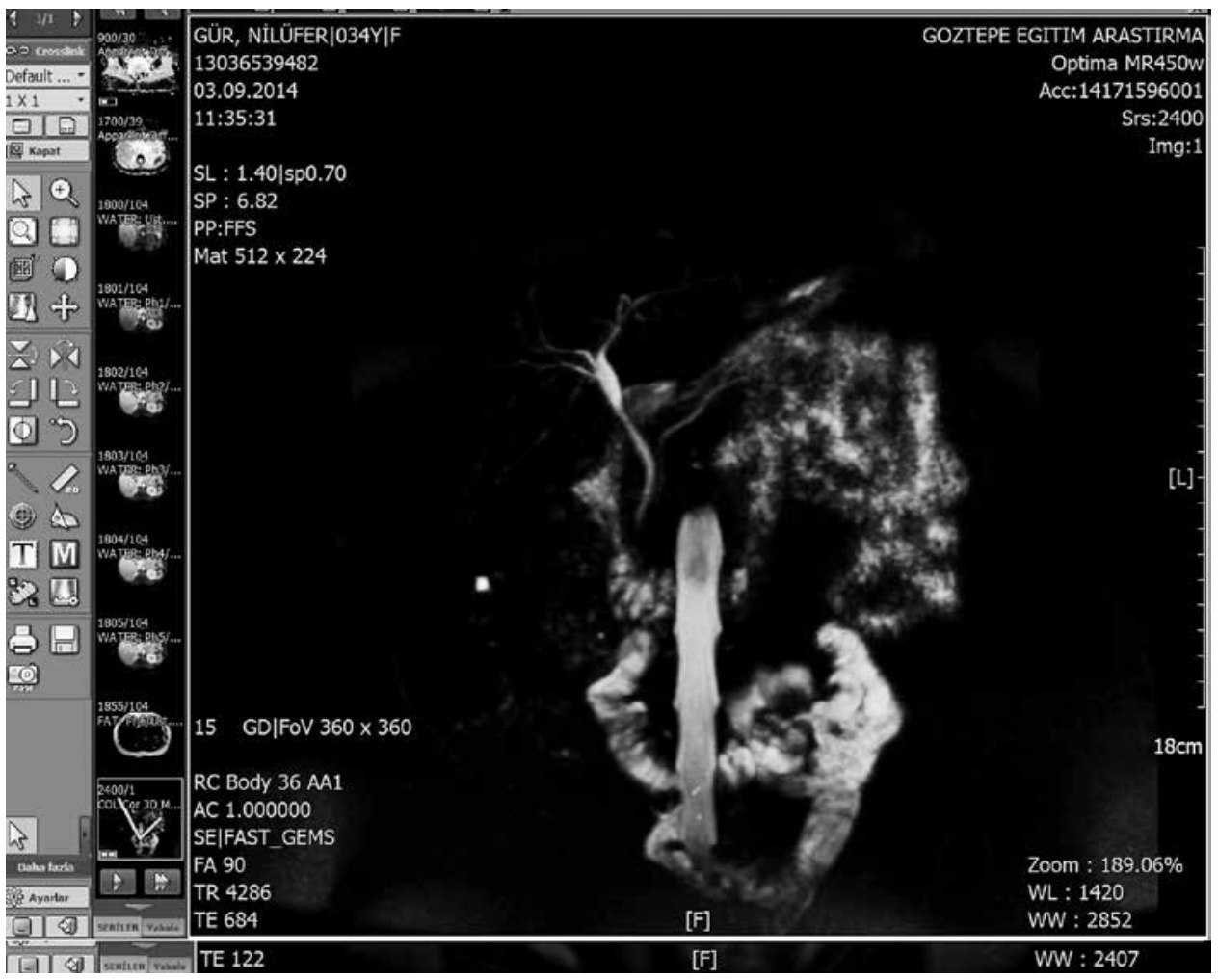

Figure 2 - The magnetic resonance cholangiopancreatography showed the absence of gallbladder.

gallbladder, cystic duct or cystic artery. In order to prevent biliary tract injury, we decided to complete operation for further diagnostic investigation. The patient was discharged on the first postoperative day. The MRCP showed the absence of gallbladder (Figure 2). The patient underwent medical treatment with complete control of symptoms.

\section{Discussion}

GA is an extremely rare condition with an incidence of $0.007-0.0027 \%$. Several studies have revealed a strong familial association with this condition. Any defect in the developmental process leads to the agenesis of gallbladder. This anomaly is transmitted as non-sex linked trait with variable penetration the actual incidence is not known (Gilbert, 1997; Gotohda et al., 2000).

The gallbladder develops from the caudal part of the hepatic diverticulum in the fourth week of embryonic life. There are two theories regarding non-development of the gallbladder. According to one theory, the hepatic diverticular bud of the foregut fails to develop properly into gallbladder and cystic duct. The other theory 
holds that, following solid-phase development, there is a failure of recanalization of cystic duct and gallbladder. Isolated GA results when the cystic bud does not develop (Bennion et al., 1988).

GA is associated with other malformations in several systems in 40-65\% (Zheng et al., 2010). In a review of autopsies in 29 cases, 13 had malformations in the genitourinary/reproductive system, 8 had cardiovascular and skeletal system malformations, 5 had abnormalities in the anterior abdominal wall and in the remaining 3 cases the GA was alone (Muguruma et al., 2001). There may be a familial tendency. The GA is associated with congenital syndromes as cerebrotendinous xanthomatosis and the G-syndrome, Klippel-Feil syndrome and trisomy 18 . Some authors reported GA as a result of thalidomide therapy. GA may be inherited with a non-sex-linked heredity, with several familial cases observed, including across two generations (Cabajo Caballero et al., 1997).

GA itself has no characteristic symptomatology. Clinically, 3 groups of GA presentation were reported by Bennion et al. in 1988:1) Multiple foetal anomalies $(15-16 \%)$ : These patients invariably die in the perinatal period due to associated anomalies and GA was only recognized at autopsy. Most frequently encountered were cardiovascular, gastrointestinal, genitourinary, anterior abdominal wall, and central nervous system anomalies. In this group, GA is only a trivial anomaly. 2) Asymptomatic group (35\%): GA was discovered either at autopsy, at laparotomy for unrelated diagnosis or by screening the family members of patients known to have GA. These patients do not have symptoms of the biliary tract. 3) Symptomatic group (50\%): This major group presents in the $4^{\text {th }}$ or $5^{\text {th }}$ decades and this is usually an isolated anomaly. In the symptomatic group, common signs are chronic right upper quadrant pain $(90 \%)$, dyspepsia $(30 \%)$, nausea and vomiting $(66 \%)$, fatty food intolerance (37\%), and jaundice (35\%) (Malde, 2010). The possible mechanisms of symptoms include primary duct stone, biliary dyskinesia or non-biliary disorders. Biliary dyskinesia may be due to a spasm of the Oddi sphincter and is associated with increased pressure in the common bile duct. Possible causes of the pain in GA include biliary dyskinesia, adhesions in the gallbladder fossa, or periportal adhesions. The lysis of these adhesions at operation is the main cause that this pain is resolved postoperatively (Chan et al., 1997).

The use of imaging modalities in order to diagnose GA has some advantages and disadvantages that should be kept in mind Ultrasonography is the method of choice for the diagnosis of cholelithiasis or common bile duct stones with a sensitivity of $95-98 \%$. Shadowy opacities misdiagnosed as stones can be due to intestinal gas artefacts or duodenum, subhepatic peritoneal folds (O'Sullivan et al., 1987).

MRCP (magnetic resonance cholangiopancreatography) is a non-invasive and well demonstrated imaging method in the evaluation of the biliary tract. As it does not require contrast material to visualize the bile, it is not compromised by biliary 
stasis. It can then demonstrate an excluded and/or ectopic gallbladder. Preoperative MRCP should be considered in cases in which ultrasound suggests non-visualization of the gallbladder before any decision to operation.

\section{Conclusion}

GA is a very rare condition with no specific symptoms. It is associated with malformations of other systems and it seems that there is a "familiar tendency".

In conclusions, GA should be kept in mind whenever the gallbladder is improperly visualized in routine imaging methods in patients with biliary-type pain.

MRCP technique may not yet replace ultrasound as the gold standard of acute gallbladder imaging but it revealed an ideal complementary study to inconclusive ultrasonographic studies. The correct preoperative diagnosis of GA is fundamental to avoid a needless surgical exploration, which might be risky.

When laparoscopic surgery is done, further surgical procedures should be abandoned in order to prevent injuries and the patients must undergo postoperative investigation.

\section{References}

Adusumilli, S., Siegelman, E. S. (2002) MR imaging of the gallbladder. Magn. Reson. Imaging Clin. N. Am. 10(1), 165-184.

Bennion, R. S., Thompson, J. E. Jr., Tompkins, R. K. (1988) Agenesis of the gallbladder without extrahepatic biliary atresia. Arch. Surg. 123, 1257-1260.

Cabajo Caballero, M. A., Martin del Olmo, J. C., Blanco Alvarez, J. I., Atienza Sanchez, R. (1997) Gallbladder and cystic duct absence. An infrequent malformation in laparoscopic surgery. Surg. Endosc. 11, 483-484.

Chan, F. L., Chan, J. K., Leong, L. L. (1997) Modern imaging in the evaluation of hepatolithiasis. Hepatogastroenterology 44, 358-369.

Gilbert, S. F. (1997) Developmental Biology, $5^{\text {th }}$ Ed. Sinauer Associates Inc., Sunderland.

Gotohda, N., Itano, S., Horiki, S., Endo, A., Nakao, A., Terada, N., Tanaka, N. (2000) Gallbladder agenesis with no other biliary tract abnormality: report of a case and review of the literature. J. Hepatobiliary Pancreat. Surg. 7(3), 327-330.

Latimer, E. O., Mendez, F. L., Hage, W. J. (1947) Congenital absence of gallbladder. Report of three cases. Ann. Surg. 126, 229-242.

Malde, S. (2010) Gallbladder agenesis diagnosed intra-operatively: a case report. J. Med. Case Reports 4, 265.

Muguruma, N., Okamura, S., Ichikawa, S., Tsujigami, K., Suzuki, M., Nakasono, M., Kusaka, Y., Tadatsu, M., Okita, Y., Ito, S. (2001) Asymptomatic case of congenital absence of gallbladder. J. Med. Invest. 48, 118-121.

O'Sullivan, J., O'Brien, P. A., MacFeely, L., Whelton, M. J. (1987) Congenital absence of the gallbladder and cystic duct: nonoperative diagnosis. Am. J. Gastroenterol. 82, 1190-1192.

Richards, R. J., Taubin, H., Wasson, D. (1993) Agenesis of the gallbladder in symptomatic adults. A case and review of the literature. J. Clin. Gastroenterol. 16(3), 231-233.

Waisberg, J., Pinto, P. E. Jr., Gusson, P. R., Fasano, P. R., de Godoy, A. C. (2002) Agenesis of the gallbladder and cystic duct. Sao Paolo Med. J. 120(6), 192-194.

Wilson, J. E., Deitrick, J. E. (1986) Agenesis of the gallbladder: case report and familial investigation. Surgery 99(1), 106-109.

Zheng, X.Y., Shen, G. L., Sun, X. D., Huang, D. S. (2010) Agenesis of the gallbladder with adipose tissue in the gallbladder fossa. Hepatogastroenterology 57(98), 212-214. 